\title{
Study on Network Structure Characteristics of Boxers' Physical Fitness System From the Perspective of Complex Network
}

\author{
Hui Zhang ${ }^{1}$, Qingmin Fan ${ }^{2, *}$
}

\author{
${ }^{1}$ Sports department, Beijing Institute of Technology, Beijing 100081, China \\ ${ }^{2}$ Sports Coaching College, Beijing Sport University, Beijing 100084, China \\ "Corresponding author. Email: 44560304@qq.com
}

\begin{abstract}
This paper analyses the complex relationship and structural characteristics of the indicators of male boxers' strength and conditioning system from the perspective of network science. The paper also explores the core indicators of the system under different dimensions. In it, a test index system was established according to the physical characteristics of boxing. Based on the test results, the network model of the physical system of male boxers was established and the network attributes were calculated. Results: 1) There are 156 correlations among the 43 indicators of the strength and conditioning system of men's boxing. The lowest correlation is 1 , the highest is 15 , and the average is 6.6383. 2) Any indicator in the physical network can affect another indicator through an average of 2.8032 edges. The clustering coefficient of the network is 0.6075 , which indicates that the network connection is very close. 3) The maximum strength of weight and bench press are the core indicators of the strength and conditioning system of male boxers under the four central dimensions. 4) The network has obvious structural division with the module value of $Q=0.45$. The network consists of two large and four small sub-networks. The indicators of the strength and conditioning system of male boxers vary according to their attributes, thus having different impact on the whole system. These indicators do not function independently based on their different characteristics. Instead, they correlate with each other and have influence on each other as an integration. This will help find out method leading to higher level of physical performance of male boxing athletes.
\end{abstract}

Keywords: Male, boxing, physical fitness, structure, network science

\section{INTRODUCTION}

With the development of science and technology, people have a deeper understanding of the physical training. In practice, some advanced modern training ideas and methods that incorporate new ideas and techniques have also been introduced. The traditional training theory restored the original integrated strength and conditioning to the subordinates of strength, speed, endurance, flexibility, sensitivity and shape and function (Ross, $\mathrm{J}$ et al. 2009). This restoration can make people understand different roles of strength and conditioning and their contributions in the physical movement process more easily. Based on this understanding. The restoration helps continue to dissect a certain ability on the existing basis and simplify the physical training as much as possible. Simplicity. This method of restoration is scientific and has been proved to be effective in both physical training theory and practice, and has achieved very good results. However, the traditional concept of strength and conditioning classification has been challenged in an unprecedented way, especially in the training and competition practice of high-level athletes. Given the complexity of athletes' strength and conditioning and the control of factors affecting physical ability, the traditional classification of strength and conditioning no long works. Given the increasing nonlinearity, uncertainty and disordered complexity, the traditional strength and conditioning classification theory gradually loses its explanatory and predictable function (Lewis, 2009).

From the perspective of complexity science, strength and conditioning classification is essentially the division of the overall ability in the form of zero-removal and from the performance of external static capabilities. The classification views strength and conditioning in the linear causal relationship between ability and performance, over-emphasizing the sub-capabilities of strength and conditioning. The classification uses a simple paradigm to measure the complexity of strength and conditioning, and to weaken the interaction and synergy between sub-capabilities and capabilities respectively. The overall attributes of strength and conditioning and its level of performance are not a simple combination of the attributes and functions of each sub-capacity, but a higher level physical performance that emerges from the interactions and synergy of sub-capacities. Although people have realized the complexity of the strength and conditioning 
including the integrity of the sub-capacities and the complex system, many problems fails to be solved due to the limitations of science and technology. With the development of system complexity, the original theoretical interpretation of complex systems can use modern computer technology to realize the calculation of system structure and the visualization of system relationships, and gradually a new discipline, the network science, will be formed. Network science, as an emerging discipline, is a new method for exploring complex problems and is also a quantitative description tool(Bashan, et al.,2012). It provides us a new perspective and a new scientific research method to understand the complexity of athletes' strength and conditioning. At present, this new method is needed in the field of sports science to understand the attributes of athletes' strength and conditioning system. The data-based quantitative description method is needed to discover the core elements of the structure in the physical energy system, so as to better understand the athletes' strength and conditioning level, to grasp the key aspect of strength and conditioning training, to have better control of important nodes, to optimize the strength and conditioning structure and to better stimulate human body's potential. This new method will have a greater demand for athletes' strength and conditioning.

This study uses the methodology of network science to analyze the influence of the system's structure on the system's function, reveal the mechanism of physical network structure, calculate and analyze the attributes of network topology and find out the core nodes in the network through quantitative description, divide community structure and visualize the network data. The project has important theoretical and practical significance

Table 1 Test indicators classification for enriching the theoretical system of athletes' strength and conditioning, improving the understanding physical fitness and promoting the strength and conditioning of boxers.

\section{PARTICIPANTS}

A total of 47 athletes participated in the test, including China's national boxing team and the members of men's boxing team of Beijing Sport University. The average training period of the athletes is $3.96 \pm 2.41$ years, their average age is $21.13 \pm 1.3$ years old, their average height is $178.09 \pm 5.75 \mathrm{~cm}$ and their average weight is $70.87 \pm 13.89$ $\mathrm{kg}$.

\section{PROCEDURES}

Indicator construction: Based on the extensive reading of the strength and conditioning related to boxing, the paper deeply analyzes the physical characteristics of boxing and generally establishes a test index system for the strength and conditioning system of boxers. Questionnaire survey was conducted on national team's boxing coaches and experts in the strength and conditioning field. After modifying the test indicators according to experts' opinions, the test index system of boxing athletes' strength and conditioning was finalized (Table 1), including a total of 43 detail indicators under the 3 categories of body shape, body function and physical quality.

\begin{tabular}{|c|c|}
\hline categories & Indicators \\
\hline $\begin{array}{l}\text { Body } \\
\text { shape }\end{array}$ & $\begin{array}{l}\text { Height, body weight, Inter-finger distance, Shoulder width, waistline, bust, neck } \\
\text { circumference, Sitting body flexion, Upper arm circumference tense, Body fat } \\
\text { percentage }\end{array}$ \\
\hline $\begin{array}{l}\text { Physical } \\
\text { function }\end{array}$ & $\begin{array}{l}\text { White blood cell, Red blood cell, Hemoglobin, Creatine Kinase, Testosterone, } \\
\text { Cortisol, Blood Lactic Acid, Mean Power, Peak Power, VO2 Max, Heart Rate }\end{array}$ \\
\hline $\begin{array}{l}\text { Physical } \\
\text { quality }\end{array}$ & $\begin{array}{l}100 \text { meters running, } 30 \text { meters running, Average response time of hitting } \\
\text { sandbag, Shortest reaction time to hit the sandbag, Left straight punch maximum } \\
\text { strength, Right straight punch maximum strength, Left upper hook punch } \\
\text { maximum strength, Right upper hook punch maximum strength, Left swing } \\
\text { maximum strength, Right swing maximum strength, } 30 \text { punch total power, The } \\
\text { explosive power of unit weight, Strike strength per kilogram of body weight, } 15 \\
\text { seconds to hit the total power of sandbags, Deep squat maximum weight, Bench } \\
\text { press maximum weight, Hard draw maximum weight, Number of skipping, } \\
\text { Quadrant jump, Cross run, T-test, } 20 \text { meters shuttle run }\end{array}$ \\
\hline
\end{tabular}

Indicator measurement: 47 boxing athletes were tested according to strength and conditioning related indicators. The test was organized and implemented by the Beijing
Sport University and experienced boxing teachers of the university. All tests were completed at the Beijing Sport University's Key Laboratory of Strength and conditioning 
Training. To ensure the objectivity of the test data, all the athletes were tested 3 times, and the average was taken as the final score.

Strength and conditioning network model construction: This study combines the theory and practice of network science and turn the male boxer's strength and conditioning system into a abstract and complex network. The 43 indicators selected according to the strength and conditioning system are "nodes" in the network complexity. The correlation between the indicators is identified as the "edge" of the network complexity. Then, analysis of attributes related to the network complexity can be carried out. When determining the "edge" of the network, the correlation coefficient $\mathrm{R}>0.3$ between the indicators is taken as the standard and the correlation of coefficient also needs to be taken into consideration. Two nodes can form an "edge" only when the correlation coefficient is $\mathrm{R}>0.3$ between the two indicators and the correlation is $\mathrm{P}<0.05$. A node with a side edge is marked as 1 , otherwise it is marked as 0 , thereby forming a data matrix containing only 1 and 0 . The network attribute is calculated by Matlab2015a and the network graphics are processed by Gephi.

\section{RESULTS}

\subsection{Network Structure Model of Strength and Conditioning System of Male Boxers}

Based on the processed data from the above-mentioned male boxers' strength and conditioning text, the network structure model of the boxer's strength and conditioning system is constructed according to the practice method of network science. The network model of the strength and conditioning system of the male boxer is shown in Figure 1. The figure shows the network relationship and network structure of male boxers' strength and conditioning indicators. The whole network consists of two large sub-networks. The relationship between indicators is shown by the connection line in the figure. The degree of the indicator in the network is represented by the size and color of the dot. The larger the dot area is, the more connections are with other indicators in the network, the stronger the correlation is, and the greater the role the dot plays in the network. Different colors represent different degrees.

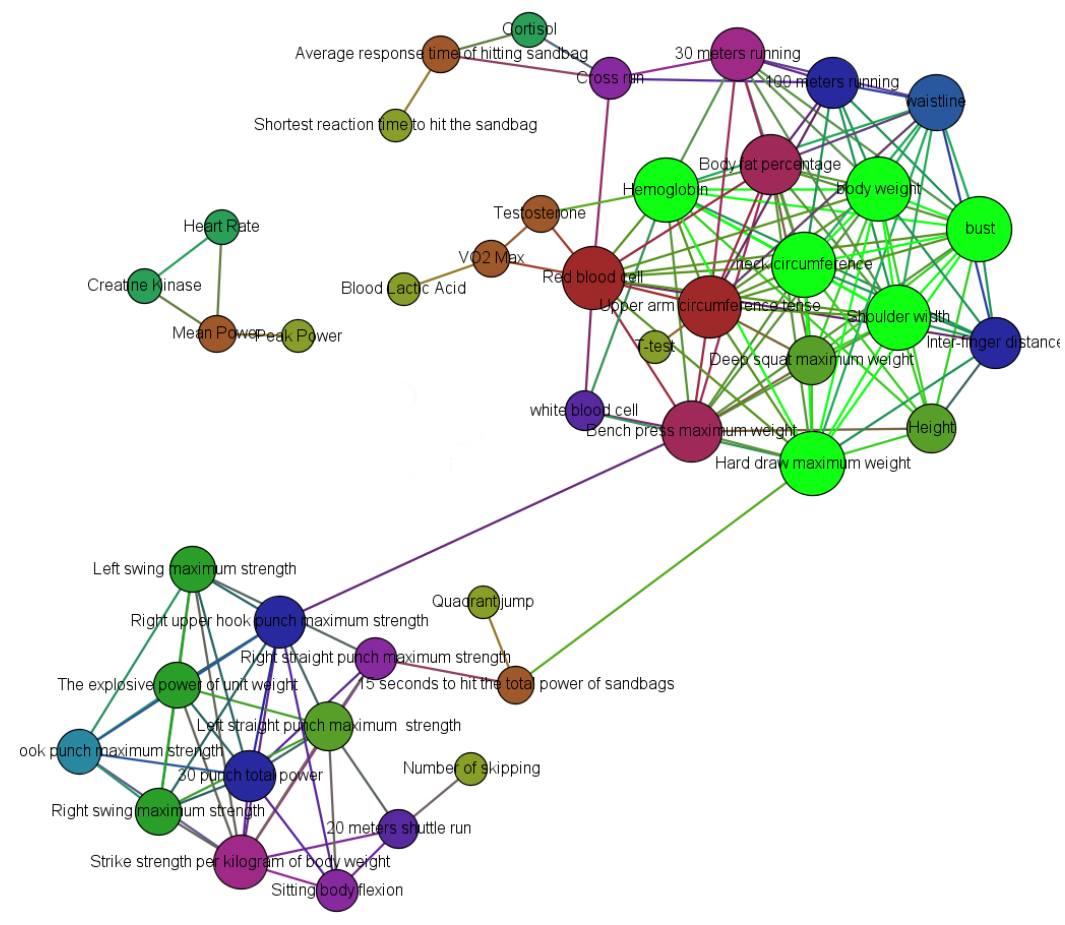

Figure 1 Network structure of physical fitness system of male boxers 


\subsubsection{Male boxers' strength and conditioning degree and its distribution in the network}

In the strength and conditioning network model, degree reflects the number of connected edges between indicators in the network. The greater the degree is, the more the connection are between indicators and the stronger the connectivity is. This reflects the important role of indicators in the network. Given the large number of indicators, only the first 10 indicators are listed and the ranking are shown in Table 2.

Table 2 Strength and conditioning index node degree distribution table

\begin{tabular}{ccc}
\hline NO & Index & Degree \\
\hline 1 & body weight & 15 \\
2 & Shoulder width & 15 \\
3 & bust & 15 \\
4 & neck circumference & 15 \\
5 & Hemoglobin & 15 \\
6 & Hard draw & 15 \\
7 & Upper arm circumference tense & 14 \\
8 & Red blood cell & 14 \\
9 & Body fat percentage & 13 \\
10 & Bench press & 13 \\
\hline
\end{tabular}

\subsubsection{The average shortest path in the male boxers' strength and conditioning network}

The average shortest path in the network is defined as the average distance between any two nodes and is used to reflect the connections between different elements in the system. The calculation shows that the average shortest path length between indicators nodes is 2.8032 .

\subsubsection{Clustering coefficient of male boxers' strength and conditioning network}

The clustering coefficient is within $0 \leq \mathrm{C} \leq 1$. The closer the figure is to 1 , the closer the connection of indicator node is. When the coefficient is equal to 1 , it means that all the indicator nodes in the network are connected. The closer the figure is to 0 , the sparser the connection is. If it is equal to 0 , it means that none of the indicators nodes are not connected to each other, or they are isolated to each other. The calculation shows that the clustering coefficient of the network is 0.6075 and the clustering coefficient value is big according to the topological attribute of the network. This, together with the network's average shortest path length of 2.8032, shows that the male boxer' strength and conditioning system is an interrelated and closely connected integration.

\subsection{The Centrality of Male Boxers' Strength and Conditioning System}

Centrality analysis is a quantitative method to analyze what role the indicators play in the system and the method is also the basis for identifying important indicators in the system. Due to the complexity of the structure and connection of the system itself, the importance of each indicator under different attributes [4] cannot be fully reflected with singe degree value. Therefore, four calculation methods including centrality, betweenness centrality, closeness centrality and eigenvector centrality are needed to analyze of the importance of various indicators in the network. Also, the core indicators in line with the characteristics of the network can be found in a comprehensive way.

\subsubsection{Ranking of the importance of the indicators of male boxers' strength and conditioning system under the degree centrality dimension}

The centrality of the degree is mainly to locate the node with a large degree value in the network. The greater degree indicates the greater value of an indicator, a stronger indicator association and therefore greater importance of the indicator in the system. The results of the analysis of the centrality of the system are shown in Table 2. Among the top 10 indicators, 6 are about body shape, 2 are about body function and 2 are about physical quality. It can be seen that these 10 body shape indicators are the core indicator nodes in the system under the degree centrality dimension.

\subsubsection{Ranking the importance of indicators of the strength and conditioning system under the betweenness centrality dimension}

The betweenness centrality is a calculation method based on the length of the path between nodes. The more shortest path passing a node there are in the network, the more important this node is. Nodes with higher betweenness centrality are often referred to as "bridge nodes". In the system, the value of "bridge nodes" is not necessarily big but it functions as a bridge in a transportation hub, playing an important connection and transition role [4]. The ranking of indicators of the system under the betweenness 
centrality dimension are shown in Table 3 . Different from the ranking under the centrality degree dimension, there are 6 physical quality indicators, 3 physical function indicators and 1 body shape indicator among the top ten indicators under the betweenness centrality dimension.

Table 3 Ranking of the importance of the indicators of strength and conditioning system under the betweenness centrality dimension

\begin{tabular}{lcc}
\hline NO & Index & Betweenness \\
\hline 1 & Bench press & 31 \\
2 & Right upper hook punch & 29 \\
3 & Red blood cell & 21 \\
4 & Cross run & 12 \\
5 & Hard draw & 10 \\
6 & 15 seconds to hit the total power & 8 \\
7 & Upper arm circumference tense & 5 \\
8 & VO2 Max & 4 \\
9 & 20 meters shuttle run & 4 \\
10 & Average response time of hitting sandbag & 4
\end{tabular}

\subsubsection{Ranking of the importance of indicators of the strength and conditioning system under the closeness centrality dimension}

Closeness centrality is also a method of importance ranking based on the path length. Unlike betweenness centrality dimension, closeness centrality ranks the importance of nodes by calculating the average length path

Table 4 Ranking of the importance of indicators of the strength and conditioning system under the closeness centrality

\begin{tabular}{lcc}
\hline NO & Index & Closeness \\
\hline 1 & Bench press & 17 \\
2 & Red blood cell & 17 \\
3 & Hard draw & 17 \\
4 & body weight & 17 \\
5 & Shoulder width & 17 \\
6 & bust & 17 \\
7 & neck circumference & 17 \\
9 & Hemoglobin & 17
\end{tabular}

from an indicator to the others in the network. The closeness centrality calculation can be free from the influence of some nodes degree in the network. The shorter the average path of one node from other nodes, the stronger the closeness centrality of the node is. The ranking of importance under the closeness centrality dimension is shown in Table 4. Among the top ten indicators, there are 6 body shape indicators 2 physical quality indicators and 2 body function indicators. 
Table 4, cont

\subsubsection{Ranking of the importance of indicators of the strength and conditioning system under the eigenvector centrality dimension}

The evaluation basis of the eigenvector centrality indicators not only considers the number of neighboring indicators connected to it, but also consider the quality and

Table 5 Ranking of the importance of each index of the strength and conditioning system under the eigenvector centrality dimension

\begin{tabular}{ccc}
\hline NO & Index & Eigenvector \\
\hline 1 & body weight & 42 \\
2 & Shoulder width & 42 \\
3 & bust & 42 \\
4 & neck circumference & 42 \\
5 & Hemoglobin & 39 \\
6 & Hard draw & 39 \\
7 & Upper arm circumference tense & 38 \\
8 & Body fat percentage & 38 \\
9 & Bench press & 34 \\
10 & Red blood cell & 34 \\
\hline
\end{tabular}

\subsection{Male Boxers' Strength and Conditioning Network Community Structure}

The community structure is a complexity network composed of several "groups". The network nodes inside each "group" are connected very closely, but the connections between the sub-groups are relatively sparse. By dividing the community of complexity networks, we can better understand the clustering of nodes with the same importance of neighboring indicators in the network and to quantify the importance of the system from two perspectives. The ranking of the importance of indicators of male boxers' strength and conditioning system under the eigenvector centrality is shown in Table 8.Among the top ten indicators in the table, there are 6 body shape indicators, 2 physical function indicators and 2 physical quality indicators. attributes in the network, go into the detail to better understand the network's functions, and also understand the hierarchical structure of the network. This helps provide a better understanding of the structure of the actual system and also a basis for a better control of the system. Based on the calculation of the community structure of the network, the network can be divided into two large communities and there are small communities consisting of two to four nodes. The visualized community structure is shown in Figure 2. 


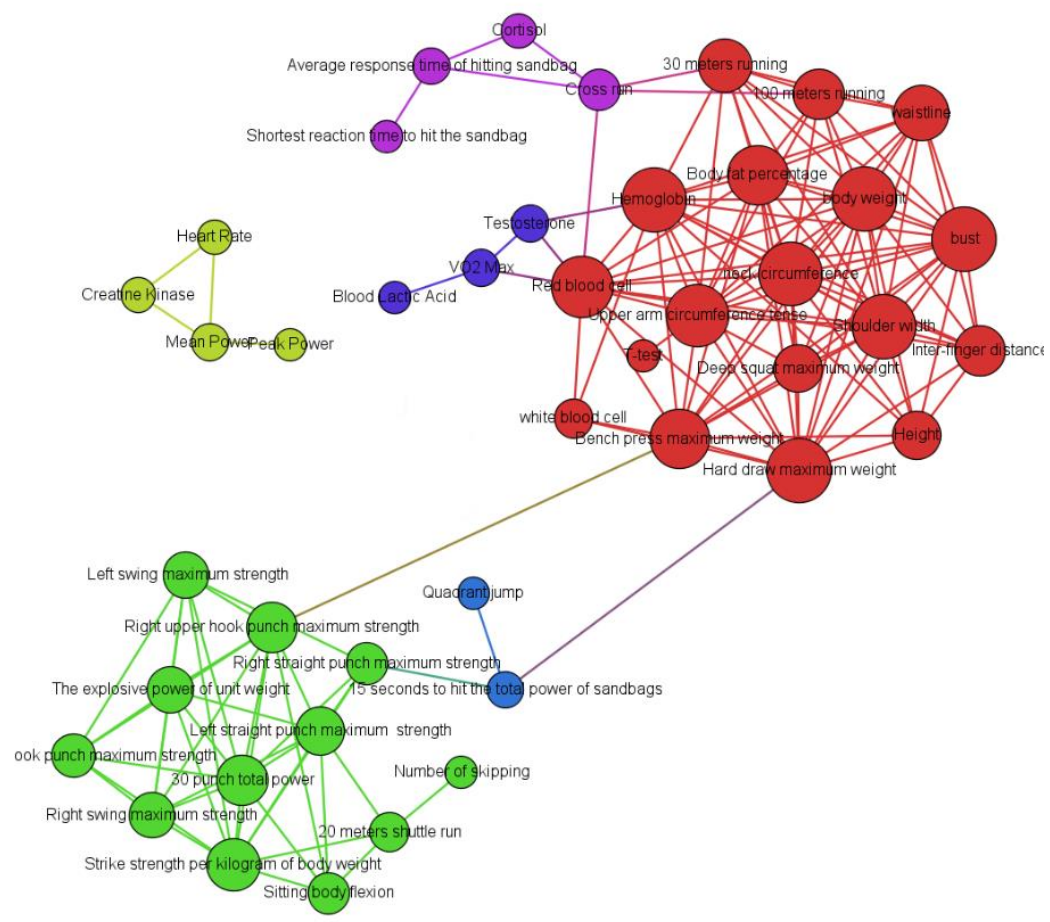

Figure 2 Network community structure of physical fitness system of male boxers

\section{DISCUSSION}

\subsection{Network Structure Characteristics of Male Boxers' Strength and Conditioning System}

There are 43 nodes with 156 joint edges in the male boxers' strength and conditioning system. The minimum value is 1 and the maximum is 15 with the average value of 6.6383. This shows that there are 156 associations among the 43 indicators in the male boxers' strength and conditioning system. The indicators with the lowest correlation degree only have one correlation with another indicator. The largest indicator is of 15 value degree with the average degree of 6.6383 . This reflects that the indicators in the system do not exist independently, but play their respective roles in strong connection. Each indicator plays a different role in the whole system according to its different attributes. The greater the value of the indicator, the greater the role it play in the network and the stronger the synergy it has with other indicators.

The indicators in the physical system network are interrelated. Given the different attributes and roles of indicators, the path lengths between indicators are also different. The average shortest path length in the male boxers' strength and conditioning system of is 2.8032 , which means that one indicator in the system can affect another one through an average of 2.8032 times of edging. The closer the indicators in the network are connected, the shorter the path length is, the stronger the correlation between the indicators is and the higher the level of capability is. The value of the clustering coefficient of the network is 0.6075 , which indicates that the network connection is very close and the indicators are related to each other, constituting a tightly connected system. During the training process of boxers, the indicators in the system play their respective roles in the system and influence each other.

\subsection{Centrality Analysis of Male Boxers' Strength and Conditioning System}

The value of centrality analysis is to find out the core indicators of the network under different dimensions, so as to have a better understanding of the important nodes in the training and competition practice, thereby improving the efficiency of training and promoting the overall level of athletes' strength and conditioning.

The boxing program is multi-level according to the weight. The weight compliance is the prerequisite for the athletes to participate in the competition. In the strength and conditioning category, body weight reflects the weights of various functional organs and body tissues of the human body and weight is also the basis for human body movement. The function of organs and body tissues also determines the level of exercise capacity. Shoulder width, chest circumference, neck circumference and upper arm tension circumference are indicators that reflect the athlete's body muscle fullness. They are also indicators of the athlete's strength and conditioning level. These 
indicators are ranked among the top ten of the degree of centrality and are the core indicators of the network under the body shape dimension. Hemoglobin and red blood cells are the same functional indicators. Hemoglobin mainly transmits oxygen and carbon dioxide in the human body and can buffer certain acidic substances. The level of the blood directly reflects the athlete's physical health. Red blood cells are the main medium in the blood to transmit oxygen in the body. These two functional indicators are related to the transmission of oxygen in the body, reflecting the athlete's body oxygen utilization and gas exchange capacity. Bench press and strength pull are indicators that reflect the athletes' upper body and trunk strength level and reflect the basic strength of boxing athletes, playing an important role in boxing training practice.

Under the betweenness centrality dimension, the first in the ranking is the maximum strength of the bench press. This indicator reflects the boxing athlete's punching speed, strength and upper limb stability. The boxer can develop the explosive power, speed and endurance of the boxing only when equipped with the basic strength of the upper limbs.. In the boxing movement, the upper limbs play a main role because the athletes' movement is achieved by the punching of the fists. The maximum strength of the bench press is important in the system. Among the top ten indicators, only the maximum strength of the back hook is the special quality of the boxing athletes and the others are just general indicators. The ranking under the centrality dimension indicates that these indicators are important indicators in the network playing a pivotal role of transmission, transformation and linkage.

Under the closeness centrality dimension, the first in the ranking is the maximum strength of the bench press. It can be seen that the maximum force of the bench press occupies an important position both under the centrality and the closeness centrality and plays an important role. The maximum strength of the bench press is the core indicator of male boxers' strength and conditioning system and also the core strength indicator of the boxing athlete. The maximum strength of the bench press ranks first among the 43 indicators of the system. This indicates that the average distance between this indicator and the others is the shortest. Also, this indicator have more influence on the whole system compared to others, thereby promoting the correlation among various indicators in the system, and jointly playing various roles for the strength and conditioning needs of boxing.

Ranking first among indicators under the eigenvector centrality dimension is the body weight indicator which also ranks first under the degree centrality dimension. Therefore, this indicator is a core indicator in the system of male boxers, playing an important role in the stability and functioning of the network structure. Body weight includes the weight of each component of the body and is the basis for the performance of athletes' physical attributes. In the boxing training, the control of weight is particularly important for athletes. In addition to meeting the requirements for weights, it is more important to show a higher level of physical performance based on the existing weight.

\subsection{Consideration on Male Boxers' Strength and Conditioning System}

Judging from the attributes of the system, the indicators in the system are not isolated, but are related to each other to reflect the athlete's physical needs. It is a fully connected network as an organic whole. From the calculation results, different indicators have different contributions to the system according their attributes and it can also be seen that indicators play a synergistic role in the network based on their own attributes. From the perspective of the community structure of the network, it is further verified that the male boxers' strength and conditioning system is an integration of correlated indicators. It is not that the indicators of same attributes form a sub-network, instead, it is indicators of different attributes are mutually related and form a network, reflecting the strength and conditioning needs of the boxers. Michael Boyle proposes to make training more functional and he believes functional is goal-oriented. In sports training, the goal refers to the specific goals of sports training. The functional training focuses on movements, not muscles. It does not emphasize the development of strength in a specific movement but achieving a balance between push and pull from a target perspective, the training of boxers can be divided into two parts: general goals and specific goals. The general goal of physical training is to make athletes stronger and have better physical strength. The specific goal of physical training is to make athletes' physical strength more in line with the special needs of boxing, so that the boxers' punch will be faster in speed and greater in strength. From the perspective of action, there is no kind of sport that only needs the strength of a single joint. Boxing is a complex sport that requires the strength of multiple muscle groups and joints. The process of boxing is based on a series of strengths. This is consistent with the structural attributes of the boxer's physical network. The function of the overall system needs the synergy of indicators. The physical quality is reflected through the technical actions. Therefore, in the physical training of boxing athletes, it is necessary to consider special features, design training programs according to special technical actions and clarify training objectives, thereby improving the coordination ability of athletes' physical quality, promoting the stability of the system's structure, and promoting higher level physical performance.

\section{CONCLUSION}

The elements of the strength and conditioning system of male boxing athletes are not independent from each other, but play respective roles in association and cooperation. The strength of each element exerts different functions in 
the whole system according to different attributes. Weight and bench press maximum strength are the core indicators in the system under four centrality perspectives, playing an important role in the stability and functioning of the system. At the same time, the connections between the indicators are very close and the entire system consists of two large scale and four small scale subsystems. The elements featuring various attributes in the system do not function independently, but are interrelated, synergistic, and mutually influential. This will promote the level of physical performance to make the boxing athletes more competitive.

\section{REFERENCES}

[1] Ross, J. \& Arkin, A. P. Complex Systems: From chemistry to systems biology. PNAS 106, 6433-6434, (2009).

[2] Lewis, T. G. Network Science: Theory and Applications. [524p.] (Wiley, 2009).

[3] Bashan, A., Bartsch, R. P., Kantelhardt, J. W., Havlin, S., Ivanov, P. C. Network physiology reveals relations between network topology and physiological function. Nat. Commun. 3, 702, (2012).

[4] St Clair Gibson, A. \& Noakes T. D. Evidence for complex system integration and dynamic neural regulation of skeletal muscle recruitment during exercise in humans. Br J Sports Med. 38, 797-806, (2004).

[5] Boccaletti, S., Latora, V., Moreno, Y., Chavez, M. \& Hwang, D. U. Complex networks: structure and dynamics. Phys. Rep. 424, 175-308 (2006).

[6] Martínez-Valencia M. A., González-Ravé, J. M., Santos-García, D. J., Alcaraz Ramón, P. E., Navarro-Valdivielso, F. Interrelationships between different loads in resisted sprints, half-squat $1 \mathrm{RM}$ and kinematic variables in trained athletes. Eur J Sport Sci. 14, 18-24, (2014).

[7] St Clair Gibson, A. et al. The role of information processing between the brain and peripheral physiological systems in pacing and perception of effort. Sports Med 36, 705-22, (2006).

[8] Barrat, A., Barthelemy, M., Pastor-Satorras, R. \& Vespignani, A. The architecture of complex weighted networks. Proc. Natl. Acad. Sci. USA 101, 3747-3752, (2004).

[9] Lawyer, G. Understanding the influence of all nodes in a network. Sci Rep. 5 (2015).
[10] West, G. B., Brown, J. H. \& Enquist, B. J. A general model for the origin of allometric scaling laws in biology. Science 276, 122-126 (1997).

[11] Bunde, A. et al. Correlated and uncorrelated regions in heart-rate fluctuations during sleep. Phys. Rev. Lett. 85, 3736-3739 (2000).

[12] Hegger, R., Bunner, M. J., Kantz, H. \& Giaquinta, A. Identifying and modeling delay feedback systems. Phys. Rev. Lett. 81, 558-561 (1998).

[13] Albert, R. \& Barabási, A. L. Statistical mechanics of complex networks. Rev. Mod. Physics 74, 47-97 (2002).

[14] Song, C., Havlin, S. \& Makse, H. A. Self-similarity of complex networks. Nature 433, 392 395 (2005).

[15] Dorogovtsev, S. N. \& Mendes, J. F. F. Evolution of networks. Adv. Phys. 51, 1079-1187 (2002).

[16] Williams, R. J. \& Martinez, N. D. Simple rules yield complex food webs. Nature 404, 180-183 (2000).

[17] Zhou, C. \& Kurths, J. Dynamical weights and enhanced synchronization in adaptive complex networks. Phys. Rev. Lett. 96, 164102 (2006).

[18] Bhalla, U. S. \& Iyengar, R. Emergent properties of networks of biological signaling pathways. Science 283 , 381-387 (1999).

[19] Newman, M. E. J. The structure and function of complex networks. Siam Rev. 45, 167-256 (2003). Pastor-Satorras, R. \& Vespignani, A. Epidemic spreading in scale-free networks. Phys. Rev. Lett. 86, 3200-3203 (2001).

[20] Banavar, J. R., Maritan, A. \& Rinaldo, A. Size and form in efficient transportation networks. Nature 399, 130-132 (1999).

[21] Boccaletti, S., Latora, V., Moreno, Y., Chavez, M. \& Hwang, D. U. Complex networks: structure and dynamics. Phys. Rep. 424, 175-308 (2006).

[22] Michael Boyle. New Functional Training for Sport [M]. Human Kinetics; 2nd edition, 2016:3-9.

[23] Vanessa H. Pereira, Claudio A.Gobatto, Theodore G.Lewis.et al. Computational and Complex Network Modeling for Analysis of Sprinter Athletes' Performance in Track Field Tests. Frontiers in Physiology.9, 843(2018). 